\title{
Hypernuclear weak decay studies with FINUDA
}

\section{FINUDA Collaboration}

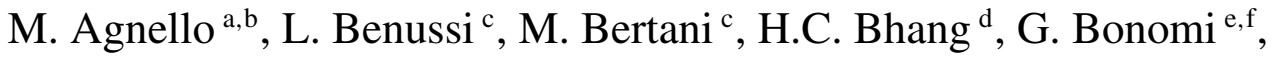

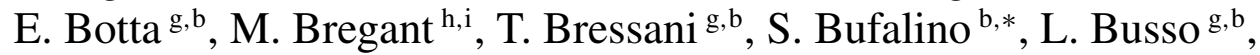
D. Calvo $^{\text {b }}$, P. Camerini ${ }^{\text {h,i }}$, B. Dalena ${ }^{\mathrm{j}}$, F. De Mori ${ }^{\mathrm{g}, \mathrm{b}}$, G. D’Erasmo $^{\mathrm{k}, \mathrm{l}}$, F.L. Fabbri ${ }^{\mathrm{c}}$, A. Feliciello ${ }^{\mathrm{b}}$, A. Filippi ${ }^{\mathrm{b}}$, E.M. Fiore ${ }^{\mathrm{k}, 1}$, H. Fujioka ${ }^{\mathrm{m}}$, P. Gianotti ${ }^{\text {c }}$, N. Grion ${ }^{\mathrm{i}}$, V. Lucherini ${ }^{\mathrm{c}}$, S. Marcello ${ }^{\mathrm{g}, \mathrm{b}}$, N. Mirfakhrai ${ }^{\text {n }}$, F. Moia ${ }^{\mathrm{e}, \mathrm{f}}$, P. Montagna ${ }^{\mathrm{o}, \mathrm{f}}$, O. Morra ${ }^{\mathrm{p}, \mathrm{b}}$, T. Nagae $^{\mathrm{m}}$, H. Outa ${ }^{\mathrm{q}}$,
\end{abstract} A. Pantaleo ${ }^{1,1}$, V. Paticchio ${ }^{1}$, S. Piano ${ }^{\text {h }}$, R. Rui ${ }^{\text {h,i }}$, G. Simonetti ${ }^{\text {k,1 }}$, R. Wheadon ${ }^{\mathrm{b}}$, A. Zenoni ${ }^{\mathrm{e}, \mathrm{f}}$

a Dip. di Scienze Applicate e Tecnologia, Politecnico di Torino, C.so Duca degli Abruzzi 24, Torino, Italy

b INFN Sez. di Torino, via P. Giuria 1, Torino, Italy

c Laboratori Nazionali di Frascati dell'INFN, via E. Fermi 40, Frascati, Italy

${ }^{\mathrm{d}}$ Dept. of Physics, Seoul National Univ., 151-742 Seoul, South Korea

e Dip. di Ingegneria Meccanica e Industriale, Università di Brescia, via Valotti 9, Brescia, Italy

${ }^{\mathrm{f}}$ INFN Sez. di Pavia, via Bassi 6, Pavia, Italy

g Dip. di Fisica, Università di Torino, via P. Giuria 1, Torino, Italy

${ }^{\mathrm{h}}$ Dip. di Fisica, Università di Trieste, via Valerio 2, Trieste, Italy

i INFN Sez. di Trieste, via Valerio 2, Trieste, Italy

j CEA, Irfu/SACM, Gif-sur-Yvette, France

${ }^{\mathrm{k}}$ Dip. InterAteneo di Fisica, via Amendola 173, Bari, Italy

${ }^{1}$ INFN Sez. di Bari, via Amendola 173, Bari, Italy

${ }^{m}$ Department of Physics, Sakyo-ku, Kyoto 606-8502, Japan

${ }^{n}$ Dept. of Physics, Shahid Behesty Univ., 19834 Tehran, Iran

${ }^{\circ}$ Dip. di Fisica Teorica e Nucleare, Università di Pavia, via Bassi 6, Pavia, Italy

p INAF-IFSI Sez. di Torino, C.so Fiume 4, Torino, Italy

q RIKEN, Wako, Saitama 351-0198, Japan

Received 17 November 2011; received in revised form 26 January 2012; accepted 26 January 2012

Available online 30 January 2012

\footnotetext{
* Corresponding author. Fax: +390116707324.

E-mail address: bufalino@to.infn.it (S. Bufalino).

1 Deceased.
} 


\begin{abstract}
The FINUDA experiment has recently completed an extensive study of the weak decay of $p$-shell $\Lambda$ hypernuclei including both mesonic and non-mesonic modes. Charged mesonic decay rates have been determined based on the analysis of $\pi^{-}$kinetic energy spectra, never measured before. The study of proton spectra from non-mesonic weak decay for $p$-shell hypernuclei, both single and in coincidence with a neutron, has triggered the investigation of the two-nucleon induced $\Lambda N N \rightarrow n N N$ decay channel: its weight has been evaluated to be $\Gamma_{2} / \Gamma_{N M}=0.21 \pm 0.07_{\text {stat }}+0.02$ sys . Finally, a direct experimental evidence of the occurrence of the weak reaction $\Lambda n p \rightarrow n n p$ in nuclei is presented for the first time. Three events have been found which can be attributed to ${ }_{\Lambda}^{7} \mathrm{Li}$ and ${ }_{\Lambda}^{9} \mathrm{Be}$ two-nucleon induced non-mesonic weak decay; the kinematical analysis is discussed here.
\end{abstract}

(c) 2012 Elsevier B.V. All rights reserved.

Keywords: Hypernuclei; Mesonic weak decay; Non-mesonic weak decay; Two-nucleon induced decay

\title{
1. Introduction
}

$\Lambda$-hypernuclei are produced in the ground state or in an excited state of the $\Lambda$-particle neutron-hole configuration. If the excitation is below the particle emission threshold, the hypernucleus decays to its ground state via fast electromagnetic transitions. A $\Lambda$-hypernucleus in the ground state decays to non-strange nuclear systems through the mesonic (MWD) or non-mesonic (NMWD) weak decay mechanisms.

In MWD the $\Lambda$-hyperon decays into a nucleon and a pion in the nuclear medium, similarly to the weak decay mode in free space:

$$
\begin{array}{ll}
\Lambda_{\text {free }} \rightarrow p+\pi^{-}+37.8 \mathrm{MeV} & (\text { B.R. }=64.2 \%), \\
\Lambda_{\text {free }} \rightarrow n+\pi^{0}+41.1 \mathrm{MeV} & (\text { B.R. }=35.8 \%)
\end{array}
$$

in which the emitted nucleon carries a momentum $q \approx 100 \mathrm{MeV} / c$, for a decay at rest. The branching ratios of the channels (1) and (2) are consistent with the empirical $\Delta I=1 / 2$ rule, valid for all non-leptonic strangeness-changing processes. Inside a hypernucleus the binding energy of the $\Lambda\left(\sim 3 \mathrm{MeV}\right.$ for ${ }_{\Lambda}^{5} \mathrm{He}, \sim 11 \mathrm{MeV}$ for ${ }_{\Lambda}^{12} \mathrm{C}, \sim 27 \mathrm{MeV}$ for $\left.{ }_{\Lambda}^{208} \mathrm{~Pb}\right)$ further reduces the energy available to the final state particles. MWD is thus suppressed in hypernuclei with respect to the free-space decay due to the Pauli principle in all nuclei except for the lightest $s$-shell ones since the momentum of the emitted nucleon is by far smaller than the nuclear Fermi momentum $\left(k_{F} \sim 270 \mathrm{MeV} / c\right)$.

In NMWD the $\Lambda$-hypernucleus decays through processes which involve a weak interaction of the constituent $\Lambda$ with one or more core nucleons. The importance of such a process was pointed out just at the beginning of hypernuclear physics: Cheston and Primakoff gave a quantitative discussion of the possibility that a $\Lambda$-hyperon bound to nucleons might undergo NMWD in 1953 [1]. If the pion emitted in the hadronic vertex $\Lambda \rightarrow \pi N$ is virtual, then it can be absorbed by the nuclear medium, resulting in a non-mesonic decay of the following types:

$$
\begin{aligned}
& \Lambda n \rightarrow n n \quad\left(\Gamma_{n}\right), \\
& \Lambda p \rightarrow n p \quad\left(\Gamma_{p}\right), \\
& \Lambda N N \rightarrow n N N \quad\left(\Gamma_{2}\right),
\end{aligned}
$$


where the $\Gamma$ 's stand for the decay rates. The channels (3) and (4) are globally indicated as onenucleon induced decays, in particular by one-neutron (3) and one-proton induced decay (4). The channel (5), referred to as two-nucleon induced decay and suggested in Ref. [2], can be interpreted by assuming that the pion from the weak vertex is absorbed by a pair of nucleons ( $n p, p p$ or $n n$ ), correlated by the strong interaction. Note that the non-mesonic processes can also be mediated by the exchange of mesons more massive than the pion.

The NMWD mode is possible only in nuclei; the Q-value of the elementary reactions (3)-(5) $(\sim 175 \mathrm{MeV})$ is high enough to avoid any Pauli blocking effect, being the outgoing nucleons momenta as high as $\sim 420 \mathrm{MeV} / c$ for the one-nucleon induced process and $\sim 340 \mathrm{MeV} / c$ for a two-nucleon induced process, if the available energy is equally distributed among the final state particles; the final nucleons thus have a large probability to escape from the nucleus. Indeed, the NMWD dominates over the MWD for all but the $s$-shell hypernuclei and only for very light systems $(A \leqslant 5)$ the two decay modes are competitive.

The total decay width of a $\Lambda$-hypernucleus $\Gamma_{T}\left({ }_{\Lambda}^{A} \mathrm{Z}\right)$ is given by the sum of the mesonic and the non-mesonic decay widths:

$$
\Gamma_{T}=\Gamma_{M}+\Gamma_{N M},
$$

where the first term can be further expressed as the sum of the decay widths for the emission of negative $\left(\Gamma_{\pi^{-}}\right)$and neutral $\left(\Gamma_{\pi^{0}}\right)$ pions:

$$
\Gamma_{M}=\Gamma_{\pi^{-}}+\Gamma_{\pi^{0}},
$$

and the second term can be written as the sum of the one-nucleon $\left(\Gamma_{1}\right)$ and two-nucleon induced $\left(\Gamma_{2}\right)$ decay widths:

$$
\Gamma_{N M}=\Gamma_{1}+\Gamma_{2},
$$

with $\Gamma_{1}=\Gamma_{p}+\Gamma_{n}$. The total decay width $\Gamma_{T}\left({ }_{\Lambda}^{A} \mathrm{Z}\right)$ is expressed in terms of the hypernucleus lifetime by:

$$
\Gamma_{T}\left({ }_{\Lambda}^{A} \mathrm{Z}\right)=\hbar / \tau\left({ }_{\Lambda}^{A} \mathrm{Z}\right) .
$$

It is then clear that several observables must be measured for a complete understanding of the weak decay of hypernuclei. The final, ambitious goal of this study is to obtain information on the four-fermion, $\Delta S=1$, baryon-baryon weak interaction, which represents the first extension of the weak, $\Delta S=0, N N \rightarrow N N$ interaction to the strange baryon sector; the weak processes $\Lambda N \rightarrow n N$ (3), (4) are the only practical way to obtain such information. It is worthwhile to mention the classification, made by Block and Dalitz in [3], of the possible $\Lambda N \rightarrow N N$ transition amplitude for ${ }_{\Lambda}^{4} \mathrm{H}$ and ${ }_{\Lambda}^{4} \mathrm{He}$. We recall that the short $\Lambda$ lifetime prevents from producing hyperon targets or beams of suitable intensity and only scarce $\Lambda N$ scattering data are presently available. Moreover, the $\Delta S=1$ interactions (3) and (4) are the best candidate to allow the investigation of the parity-conserving part of the baryon-baryon weak interaction, which is completely masked by the strong component in the $N N \rightarrow N N$ reaction [4-6].

To reach this objective, a series of coordinated experiments has to be pursued. All the spurious effects, in particular the Final State Interactions (FSI) which affect the measurements in a nuclear medium, must be clearly understood and parametrized. The decay mode (5), which introduces a further complication in the elementary decay mechanism, namely the correlation among two nucleons, has too to be deeply understood and parametrized. 
This program was recently started with the measurement of some of the observables undertaken systematically in the last two decades, allowing a considerable progress in the understanding of the process, supported also by dedicated theoretical efforts (for recent reviews see [4-6]). The FINUDA experiment provided recently a remarkable contribution to the subject, in particular with the measurement of $\Gamma_{\pi^{-}} / \Gamma_{\Lambda}$ and $\Gamma_{2} / \Gamma_{N M}$ in $p$-shell hypernuclei. The present paper is organized as follows. In Section 2 we provide a short description of the FINUDA detector performances, in particular concerning the measurement of the observables described in the following. Section 3 summarizes the main results obtained so far for $\Gamma_{\pi^{-}} / \Gamma_{\Lambda}$. Section 4 summarizes the results on proton spectra from NMWD and the determination of $\Gamma_{2} / \Gamma_{N M}$. Section 5 reports the first direct experimental evidence of the weak $\Lambda+(n p) \rightarrow n+n+p$ decay.

\section{FINUDA, a detector optimized for the study of hypernuclear weak decay}

The FINUDA detector has been described in several papers [7-10] and we refer to them for details concerning the data takings, the targets and the performances of the various subdetectors. It was installed at one of the two interaction regions of the DA $\Phi$ NE $\left(e^{+}, e^{-}\right) \phi(1020)$-factory of INFN-LNF (Italy). Hypernuclei were produced by means of the strangeness exchange reaction: $K_{\text {stop }}^{-}+n \rightarrow \Lambda+\pi^{-}$, on very thin targets $\left(\sim 200 \mathrm{mg} / \mathrm{cm}^{2}\right)$ [8]. FINUDA was a magnetic spectrometer immersed in a uniform solenoidal magnetic field of $1 \mathrm{~T}$. The tracker included a combination of silicon microstrips (OSIM), low mass drift chambers (LMDC) and a stereo system of straw tubes (ST). It was optimized for the detection of charged particles featuring a momentum resolution $<1 \%$ FWHM for $\pi^{-}$of $250-290 \mathrm{MeV} / c$, corresponding to the formation of hypernuclear bound states, and an energy resolution for $p$ ranging from $1.3 \%$ to $4 \%$ FWHM for kinetic energies from $90 \mathrm{MeV}$ down to $50 \mathrm{MeV}$ [8]. Its angular acceptance was $\sim 2 \pi$ sr. OSIM and LMDC were used also for P.Id. of the charged particles. The inner FINUDA detector was an hodoscope of thin scintillator slabs (TOFINO) arranged around the beam pipe at the $\left(e^{+}, e^{-}\right)$ interaction point. An array of silicon microstrips (ISIM) was located outside TOFINO, very close to the targets, to determine the position of the $K^{ \pm}$interaction point. An array of eight target tiles was located between ISIM and the tracker. The outer FINUDA detector, called TOFONE, was a plastic $(\mathrm{CH})_{n}$ scintillator barrel of 80 elements $(10 \mathrm{~cm}$ thick) surrounding the tracker. It was also used to detect neutrons (and photons) with a flat detection efficiency of about $10 \%$ for neutrons in the energy range 15-150 MeV [11]. The particles Time-Of-Flight (TOF) was measured by the (TOFINO-TOFONE) system. Neutrons and photons are identified looking for events in which TOFONE elements are not connected to curve trajectories belonging to charged particles. The $\beta$ of the neutral particle is evaluated by the measured TOF and the corresponding base of flight. The analysis of the $\beta$ values of the neutral candidates allows to discriminate neutrons from $\gamma$ 's emitted, for instance, in the $\pi^{0}$ decay. A contamination from neutron scattering in the FINUDA apparatus is also present in the neutrals spectrum. Applying a cut on the $1 / \beta$ value it is possible to eliminate the $\gamma$ peak from the single neutron detection, but the most effective way to identify neutron from $2 \mathrm{~N}$-induced NMWD, however, is to tag them with the proton emitted in coincidence. The neutron energy resolution is $\sim 11 \%$ at $20 \mathrm{MeV}$ and $\sim 20 \%$ at $100 \mathrm{MeV}$ [9]. The global (detection plus acceptance) efficiency for neutrons is quite flat with an average value of $(5.08 \pm 0.14) \%$.

With FINUDA several reactions induced by stopped $\mathrm{K}^{-}$were studied, taking advantage of the good detection capabilities briefly outlined. Moreover impressive and unexpected results were obtained, with FINUDA, for the weak decays studies. To this purpose, pions and nucleons from reactions (1)-(5) have to be detected in coincidence with a $\pi^{-}$signaling the formation of a 

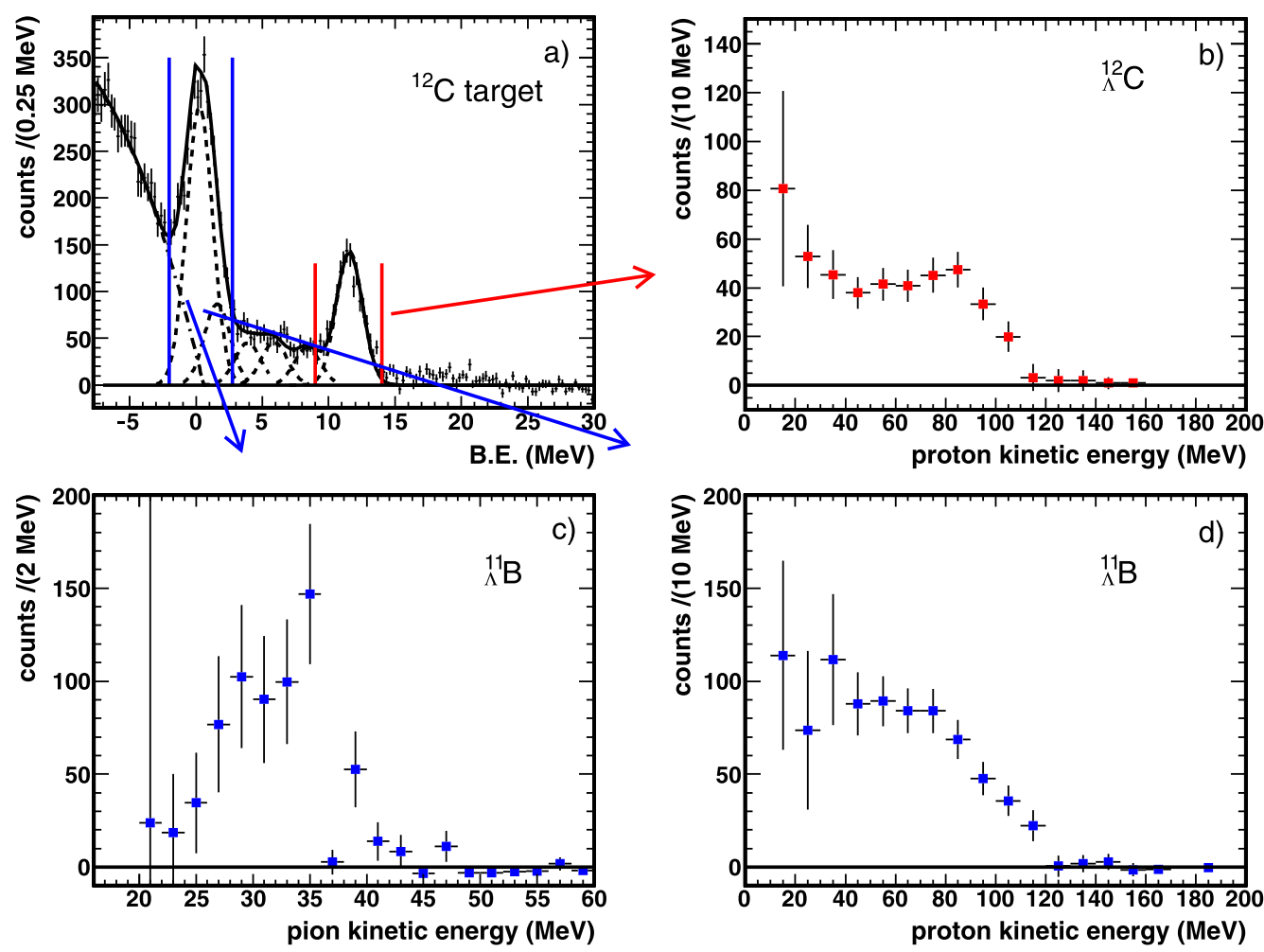

Fig. 1. (a) Binding energy spectrum of $\pi^{-}$emitted from ${ }^{12} \mathrm{C}$ targets. The spectrum is background subtracted and the dashed lines correspond to known ${ }_{\Lambda}^{12} \mathrm{C}$ energy levels; (b) energy spectrum of protons emitted in coincidence with $\pi^{-}$ from ${ }_{\Lambda}^{12} \mathrm{C}$ formation [12]; (c) energy spectrum of low energy $\pi^{-}$emitted in coincidence with $\pi^{-}$from ${ }_{\Lambda}^{11} \mathrm{~B}$ formation [7]; (d) energy spectrum of protons emitted in coincidence with $\pi^{-}$from ${ }_{\Lambda}^{11} \mathrm{~B}$ formation [8].

hypernucleus in the ground state. The quality cuts applied to $\pi^{-}$tracks in the analysis of these coincidence events were looser than the ones used from hypernuclear spectroscopy [10], with a worsening of the resolution (from $0.5 \%$ to $1 \%$ ) but with an increase of a factor 6 in the statistics. Fig. 1a shows, as an example, the binding energy (B.E.) spectrum of ${ }_{\Lambda}^{12} \mathrm{C}$ obtained with these looser cuts on the $\pi^{-}$spectrum. The two prominent peaks can be interpreted as due to the $\Lambda$ in an $s$-state, the ground state of ${ }_{\Lambda}^{12} \mathrm{C}$, and in a $p$-state. The peak at lower B.E. is mainly due to ${ }_{\Lambda}^{11} \mathrm{~B}+p$ configurations. Thus, by selecting a window in the $\pi^{-}$spectrum, corresponding to the ${ }_{\Lambda}^{12} \mathrm{C}_{\text {g.s. }}$ and ${ }_{\Lambda}^{11} \mathrm{~B}$ bound states, the spectra of protons emitted in NMWD for these two hypernuclides can be obtained (Figs. 1b and 1d) [8,12]. The same technique was used to obtain the neutron spectrum of NMWD of a well defined hypernuclide. Also the low momentum $\pi^{-}$'s from charged MWD may be detected. In this respect, for ${ }^{12} \mathrm{C}$ targets, only the ${ }_{\Lambda}^{11} \mathrm{~B}$ MWD was studied [7] and it is reported in Fig. 1c, due to the $80 \mathrm{MeV} / c$ threshold on the detection of low-energy $\pi^{-}$.

We recall that several nuclear targets of $p$-shell nuclei were used in the same data taking, and a complete systematics of the corresponding $p$-shell hypernuclei was obtained in a unique run avoiding the need of a subsequent normalization between the targets. 


\section{Measurement of $\Gamma_{\pi^{-}} / \Gamma_{\Lambda}$}

MWD is strictly forbidden in normal infinite nuclear matter, where the nucleon Fermi momentum is about $270 \mathrm{MeV} / c$, while in finite nuclei it can occur because of three important effects:

(i) in nuclei the hyperon has a momentum distribution, being confined in a limited spatial region, that allows larger momenta for the emitted nucleon;

(ii) the final pion feels an attraction by the nuclear medium, due to the $p$-wave part of the optical $\pi$-nucleus potential, which modifies the pion dispersion relation; for a fixed momentum, the pion carries an energy smaller than if it was free and the energy conservation increases the chance for the emitted nucleon to lie above the Fermi surface. Indeed, it has been shown that the pion distortion increases the MWD width by more than one order of magnitude for very heavy hypernuclei $(A \sim 200)$ with respect to the value obtained without the medium distortion [13];

(iii) at the nuclear surface the local Fermi momentum can be smaller than $270 \mathrm{MeV} / c$ and the Pauli blocking is less effective in forbidding the decay. In any case, the MWD rate rapidly decreases as the hypernucleus mass number increases.

It must be stressed that the information available before the FINUDA measurements on the charged MWD of light hypernuclei consisted almost entirely of $\Gamma_{\pi^{-}} / \Gamma_{\Lambda}$ values obtained by counting coincidences with the hypernuclear formation $\pi^{-}$, with no magnetic analysis of the decay meson; the $\pi^{-}$kinetic energy spectra were reported for ${ }_{\Lambda}^{12} \mathrm{C}$ MWD only [14]. The $\pi^{-}$energy spectra of Ref. [7], on the other hand, allow a more careful confirmation of the elementary mechanism that is supposed to underlie the decay process, through the determination of the decay rates, as well as to gain information on the spin-parity of the initial hypernuclear ground state. In this respect, the study of pion spectra from MWD can be regarded as an indirect spectroscopic investigation tool.

In particular, the ratios $\Gamma_{\pi^{-}} / \Gamma_{\Lambda}$ were obtained from the measured branching ratios, using the available $\Gamma_{\mathrm{T}} / \Gamma_{\Lambda}$ values or relying on a linear fit to the known values of all measured $\Lambda$-hypernuclei in the mass range $A=4-12$ [15]. Fig. 2 reports the values obtained by FINUDA (full (red) circles) compared with previous experimental data (full (black) triangles) and theoretical calculations (full (green) squares and full (blue) stars) for ${ }_{\Lambda}^{5} \mathrm{He},{ }_{\Lambda}^{7} \mathrm{Li},{ }_{\Lambda}^{9} \mathrm{Be},{ }_{\Lambda}^{11} \mathrm{~B}$ and ${ }_{\Lambda}^{15} \mathrm{~N}$.

Being characterized by a small Q-value, the MWD mode is strongly affected by the details of both the hypernucleus and the daughter nucleus structure. Indeed, the calculations of Refs. [20,21] evaluate the $p$-shell hypernuclei total and partial MWD decay rates by incorporating the pion final state interaction by pion-nuclear distorted waves and by describing the structure of the nuclear core with the Cohen-Kurath spectroscopic approach [23]. The authors found that for $p$-shell hypernuclei the total $\pi^{-}$decay rate is dominated by $\Lambda_{1 s} \rightarrow p_{1 p}$ transitions, while only little contributions are given by higher energy configurations of the final nuclear system, mainly through $\Lambda_{1 s} \rightarrow p_{2 s, 1 d}$ transitions.

Recently, in Ref. [22] these calculations were revisited, following the same approach, and a new sum rule was introduced to encapsulate the suppressive effect of the Pauli principle on the total and partial $\pi^{-}$decay rates. In Fig. 2, both calculations of Refs. [20,21] (full (green) squares) and Ref. [22] (full (blue) stars) are reported, for ground state spin-parity $1 / 2^{+}$for ${ }_{\Lambda}^{5} \mathrm{He}$ and ${ }_{\Lambda}^{7} \mathrm{Li}$, $5 / 2^{+}$for ${ }_{\Lambda}^{11} \mathrm{~B}$ and $3 / 2^{+}$for ${ }_{\Lambda}^{15} \mathrm{~N}$.

A good agreement holds among the FINUDA results and previous measurements (full (black) triangles), when existing, and among the FINUDA results and the theoretical calculations. The 


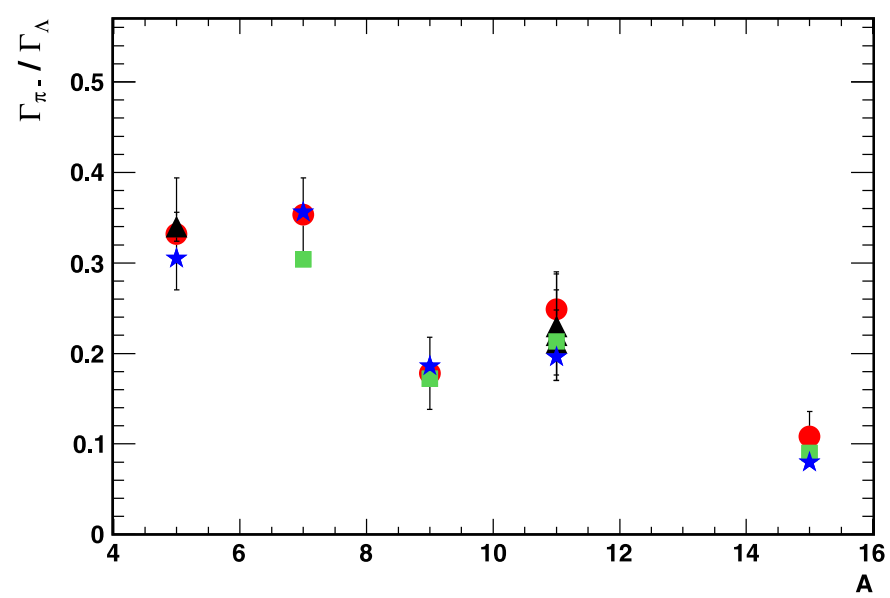

Fig. 2. The $\Gamma_{\pi}-/ \Gamma_{\Lambda}$ ratios obtained by the FINUDA experiment (full (red) circles) [7], compared with previous measurements (full (black) triangles) [16-19,14] and with theoretical calculations [20,21] (full (green) squares) and [22] (full (blue) stars). See Ref. [7] for more details. (For interpretation of the references to color in this figure legend, the reader is referred to the web version of this article.)

total decay rates for the other set of ground state spin-parity calculated in Refs. [20-22] (3/2+ for ${ }_{\Lambda}^{7} \mathrm{Li}, 7 / 2^{+}$for ${ }_{\Lambda}^{11} \mathrm{~B}$ and $1 / 2^{+}$for ${ }_{\Lambda}^{15} \mathrm{~N}$ ) are substantially lower and disagree with the experimental values; an exception is given by ${ }_{\Lambda}^{15} \mathrm{~N}$, for which the calculated values reported in Ref. [21] do not allow to fix the ground state spin-parity.

The agreement between experimental data and calculations supports the correctness of the theoretical hypotheses. It clearly indicates the possibility to evaluate typical nuclear physics observables, like the rates of the MWD of hypernuclei, starting from the knowledge of the elementary phenomenological Hamiltonian which describes the decay of the free $\Lambda$. The agreement also confirms the presence of the distortion of the outgoing $\pi^{-}$wave function due to the polarization effect of the nuclear medium and of the effectiveness of the Cohen-Kurath spectroscopic calculations in describing both the hypernucleus core and the daughter nucleus, which allow to account naturally for the strong final state shell-structure-dependence and charge-dependence of the measured decay rates.

The kinetic energy spectra of the decay pions obtained by FINUDA for MWD of ${ }_{\Lambda}^{7} \mathrm{Li},{ }_{\Lambda}^{9} \mathrm{Be}$, ${ }_{\Lambda}^{11} \mathrm{~B}$ and ${ }_{\Lambda}^{15} \mathrm{~N}$ [7] show interesting structures which can be directly related to the excitation function of the daughter nucleus as calculated by Refs. [21,20,22], allowing to determine the spin-parity configuration of the hypernucleus ground state. In Fig. 3 the $\pi^{-}$kinetic energy spectrum from ${ }_{\Lambda}^{15} \mathrm{~N}$ is showed. For instance, before the FINUDA measurement, the ground state spin of ${ }_{\Lambda}^{15} \mathrm{~N}$ was not determined experimentally. The most recent theoretical study of hypernuclear spin-dependence [24] predicts $J^{\pi}\left({ }_{\Lambda}^{15} \mathrm{~N}_{\text {g.s. }}\right)=3 / 2^{+}$, setting the $1 / 2^{+}$excited state of the ground state doublet about $90 \mathrm{keV}$ above the $3 / 2^{+}$state. The spin ordering, however, could not be determined from the $\gamma$-ray de-excitation spectra measured recently on a ${ }^{16} \mathrm{O}$ target at BNL [25]. The FINUDA measured spectrum of $\pi^{-}$supports this $3 / 2^{+}$theoretical assignment. It is thus evident that the study of the MWD spectra has strong potentialities for the determination of the ground state spin-parity for $s$ - and $p$-shell hypernuclei; for $s d$-shell strange nuclear systems, the spectroscopy of particles emitted in the decay processes can help as well in fixing the spectroscopic configuration of the ground state, although in this case the MWD branching ratios drops below $10 \%$ for these hypernuclei and the errors become consequently sizable. The MWD spectra 


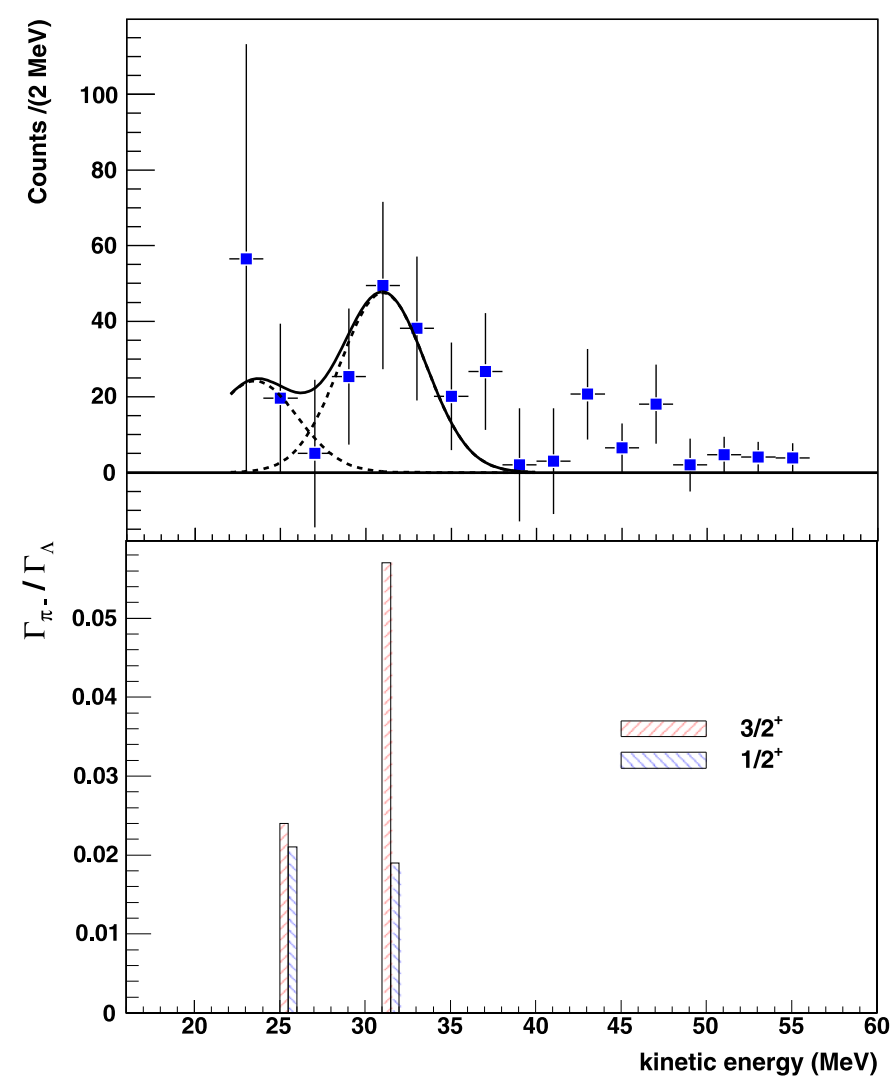

Fig. 3. Upper part: kinetic energy spectrum of MWD $\pi^{-}$for ${ }_{\Lambda}^{15} \mathrm{~N}$ after acceptance correction. The solid curve is a twoGaussian fit to the peaks in the spectrum, to compare with theoretical predictions in the lower part. Lower part: calculated major decay rates to final ${ }^{15} \mathrm{O}$ states from [22], in red bars for ${ }_{\Lambda}^{15} \mathrm{~N}$ ground-state spin-parity $3 / 2^{+}$, and in blue bars for ${ }_{\Lambda}^{15} \mathrm{~N}$ ground-state spin-parity $1 / 2^{+}$. From Ref. [7]. (For interpretation of the references to color in this figure legend, the reader is referred to the web version of this article.)

analysis has thus demonstrated its reliability as light- and medium- $A$ hypernuclei spectroscopic tool, complementary to the $\gamma$-ray spectroscopy of low-lying excited states [26], whose spin ordering cannot be determined. It represents a new version of the old technique based on the study of the decay rates and of the angular distribution of the MWD $\pi^{-}$, which allowed the ground state spin-parity determination of light hypernuclei in emulsion experiments [27-31], based on the known properties of the free $\Lambda$ weak decay.

Very recently plans for performing high resolution $(\sim 100 \mathrm{keV})$ spectroscopy of $\pi^{-}$from MWD of hyperfragments produced by electroproduction at JLab and MAMI-C on nuclear targets were put forward [32]. However, it will be difficult from such experiments to determine the spin-parity of the hypernuclear ground states, since the quantum numbers of the initial state are difficult, if not impossible, to determine.

\section{Proton spectra from NMWD and determination of $\Gamma_{2}$}

The proton energy spectra were first measured, with high statistics, in experiments at KEK $[33,34]$ for ${ }_{\Lambda}^{5} \mathrm{He}$ and ${ }_{\Lambda}^{12} \mathrm{C}$ hypernuclei, by means of a combination of TOF and total energy de- 
position. The energy loss inside the thick targets was corrected with an event-by-event analysis. The energy resolution was poor in the high energy region, especially above $100 \mathrm{MeV}$, with the consequence that the spectra could be strongly distorted. Whereas for ${ }_{\Lambda}^{5} \mathrm{He}$ an indication for a peak at $\sim 80 \mathrm{MeV}$ appeared, as expected by the occurrence of the reaction $\Lambda+p \rightarrow n+p$ in nuclei, no structure at all was reported for ${ }_{\Lambda}^{12} \mathrm{C}$. The proton spectrum with an energy threshold of 35 $\mathrm{MeV}$ showed a continuous distribution [35,36], in some agreement with theoretical calculations based on IntraNuclear Cascade (INC) model [37].

In FINUDA the proton momentum was measured by magnetic analysis, with an excellent resolution and without distortion of the spectra due to the target thickness. A further advantage of FINUDA was its lower energy threshold (15 MeV). In a first analysis proton energy spectra coming from NMWD of ${ }_{\Lambda}^{5} \mathrm{He},{ }_{\Lambda}^{7} \mathrm{Li}$ and ${ }_{\Lambda}^{12} \mathrm{C}$ were studied [12]. All of them show a quite similar shape, i.e. a peak at around $80 \mathrm{MeV}$, with a low energy rise, due to Final State Interaction (FSI) and $2 \mathrm{~N}$-induced weak decays $(\Lambda+(n p) \rightarrow n+n+p, \Lambda+(p p) \rightarrow n+p+p)$. If the low energy rises were predominantly due to FSI effect one should naturally expect that the broad peak structure at $80 \mathrm{MeV}$ (coming from clean $\Lambda p \rightarrow n p$ weak processes broadened by the Fermi motion of nucleons) were smeared out for the heavier nuclei. Concerning the $2 \mathrm{~N}$-induced NMWD, if the weak decay Q-value is shared by three nucleons, a low-energy rise may exist even for the very light $s$-shell hypernuclei. The first FINUDA data, thus, seem to indicate a substantial contribution of the two-nucleon induced NMWD.

Triggered by this observation, proton spectra for all other $p$-shell hypernuclei were studied. These spectra, shown in Fig. 4, feature a similar behavior, i.e., the presence of a structure around $80 \mathrm{MeV}$ even for ${ }_{\Lambda}^{16} \mathrm{O}$.

A technique was devised to disentangle the contribution coming from the two-nucleon induced decays, from the one-nucleon induced decays with FSI [8]. The systematics in the mass number range $A=5-16$, covered by FINUDA was exploited. Each spectrum was fitted, for a proton kinetic energy from $80 \mathrm{MeV}$ onwards, with a Gaussian function to determine, through its mean value, the energy corresponding to the maximum of the one-proton induced contribution. The spectrum was then divided in two parts, one below the mean value, with area $A_{\text {low }}$, and one above it, with area $A_{\text {high }}$. It was assumed the first part to be populated by one-proton induced decays, two-nucleon induced decays and FSI processes, while the second part has contributions from one-proton induced decays and FSI, neglecting the two-nucleon induced decays, which, above $70 \mathrm{MeV}$, accounts for only $5 \%$ of the total two-nucleon induced strength [38].

With the assumption that the FSI effects are proportional to $A$ and that the $\Gamma_{2} / \Gamma_{p}$ is constant in the $A=5-16$ hypernuclear mass range [4,5], we found [8]:

$$
\frac{\Gamma_{2}}{\Gamma_{p}}=0.43 \pm 0.25 .
$$

In order to determine $\Gamma_{2} / \Gamma_{\mathrm{NM}}$, the average of the values of $\Gamma_{n} / \Gamma_{p}$ from Ref. [40] was used. The result is:

$$
\frac{\Gamma_{2}}{\Gamma_{N M}}=0.24 \pm 0.10
$$

This value is in agreement with the theoretical predictions [39,41-46] and the latest KEK experimental results of Ref. [48].

A similar approach was used later by FINUDA to extract the $\Gamma_{2} / \Gamma_{N M}$ ratio for $A=5-16$ hypernuclei using $n p$ coincidence spectra [9]. We remind that, whereas the TOFONE was not well suited to detect one-nucleon induced NMWD due to the low detection efficiency $(\sim 10 \%)$ [9], it 

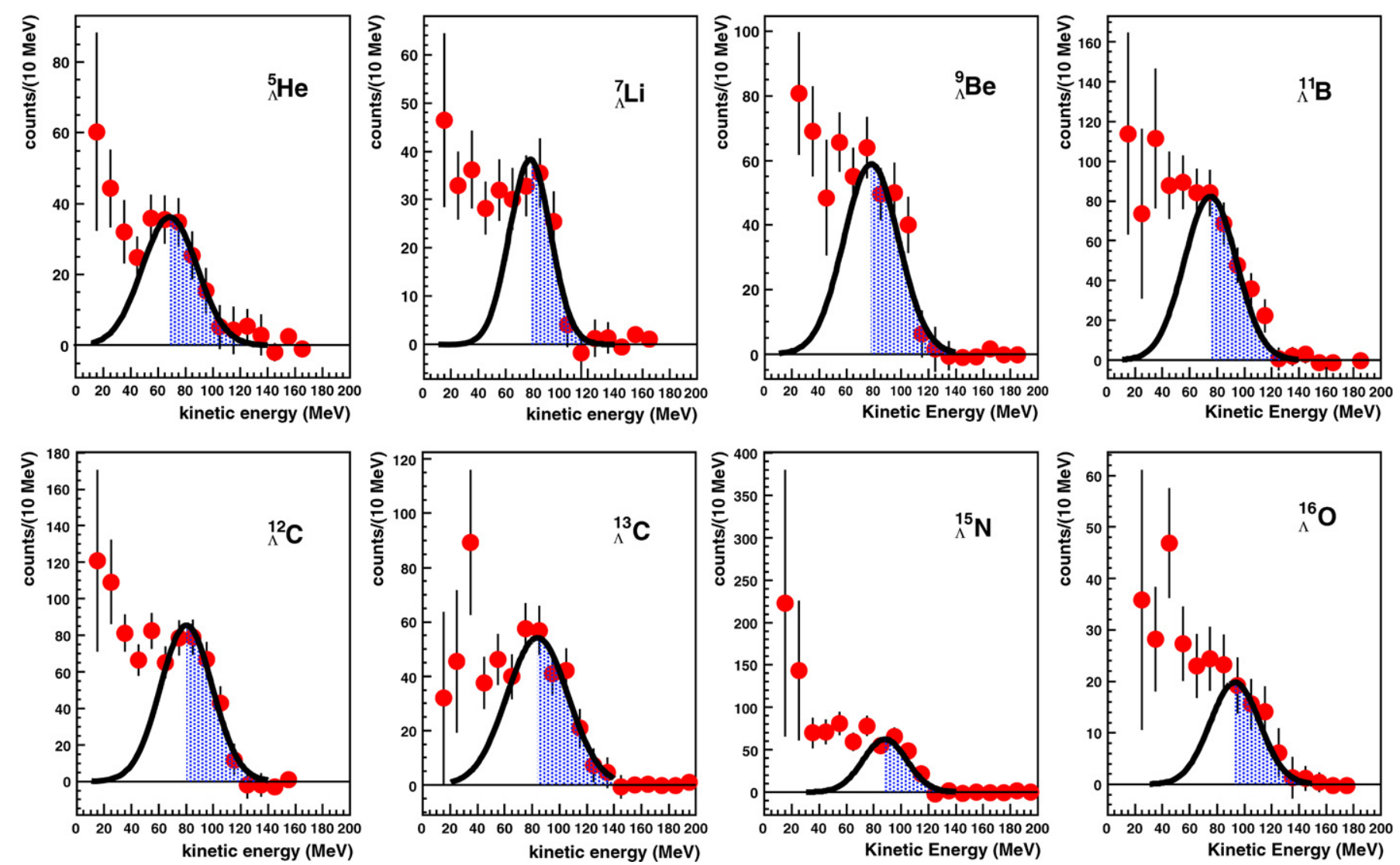

Fig. 4. Proton kinetic energy spectra from the NMWD of (from left to right): ${ }_{\Lambda}^{5} \mathrm{He},{ }_{\Lambda}^{7} \mathrm{Li},{ }_{\Lambda}^{9} \mathrm{Be},{ }_{\Lambda}^{11} \mathrm{~B},{ }_{\Lambda}^{12} \mathrm{C},{ }_{\Lambda}^{13} \mathrm{C},{ }_{\Lambda}^{15} \mathrm{~N}$ and ${ }_{\Lambda}^{16} \mathrm{O}$. The blue filled area is the spectrum area in which the two-nucleon induced NMWD is negligible. From Ref. [8]. (For interpretation of the references to color in this figure legend, the reader is referred to the web version of this article.) 
Table 1

Summary of the theoretical and experimental values of $\Gamma_{2} / \Gamma_{\Lambda}$ and $\Gamma_{2} / \Gamma_{N M}$.

\begin{tabular}{llll}
\hline Ref. & $\Gamma_{2} / \Gamma_{\Lambda}$ & $\Gamma_{2} / \Gamma_{N M}$ & Notes \\
\hline [41] & 0.23 & 0.16 & ${ }_{\Lambda}^{12} \mathrm{C}, \Gamma_{2}=\Gamma_{n p}$ \\
{$[42]$} & 0.16 & 0.16 & ${ }_{\Lambda} \mathrm{C}, \Gamma_{2}=\Gamma_{n p}$ \\
[46] & 0.25 & 0.26 & $12 \mathrm{C}, \Gamma_{2}=\Gamma_{n p}+\Gamma_{n n}+\Gamma_{p p}$ \\
BNL-E788 [47] & & $\leqslant 0.24$ & ${ }_{\Lambda} \mathrm{He}, n$ and $p$ spectra \\
KEK-E508 [48] & $0.27 \pm 0.13$ & $0.29 \pm 0.13$ & ${ }_{\Lambda}^{12} \mathrm{C}, n n$ and $n p$ spectra \\
FINUDA [8] & & $0.24 \pm 0.10$ & $A=5-16, p$ spectra \\
FINUDA [9] & & $0.21 \pm 0.07_{\text {stat }}+0.03$ sys & $A=5-16, n p$ spectra \\
\hline
\end{tabular}

was useful for the study of two- (or more) nucleon induced NMWD thanks to the full azimuthal angle coverage. We selected $\left(\pi^{-}, n, p\right)$ coincidence events with a $\pi^{-}$in the momentum region corresponding to the formation of a ground state hypernucleus, and a proton with a kinetic energy more than $20 \mathrm{MeV}$ lower than the mean value $\mu$ of the Gaussian function determined in [8], $E_{p}<(\mu-20 \mathrm{MeV})$, and an opening angle between the two emitted nucleons, $\cos \theta(n p) \geqslant-0.8$. These selections enhanced the contribution of two-nucleon induced weak decays.

Also in this case we applied the same considerations on the $A$-dependence of FSI and $\Gamma_{2} / \Gamma_{p}$ used for the study of the single proton spectra, obtaining finally:

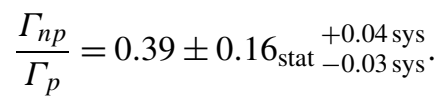

The obtained $\Gamma_{n p}$ width can be used to evaluate $\Gamma_{2} / \Gamma_{N M}$ by following the theoretical predictions of Ref. [39]; moreover, assuming again the same $\Gamma_{n} / \Gamma_{p}$ value used in (11) we found:

$$
\frac{\Gamma_{2}}{\Gamma_{N M}}=0.21 \pm 0.07_{\text {stat }}^{+0.02 \text { sys }},
$$

where the error is reduced with respect to that of Ref. [8]. The most updated theoretical evaluation [46] and experimental determinations of $\Gamma_{2} / \Gamma_{\Lambda}$ and $\Gamma_{2} / \Gamma_{N M}$ can be found in Table 1, together with information on the hypernucleus, the isospin channels considered in the calculations and on the spectra (single or in coincidence) analyzed in the experiments. All the calculations were performed within a nuclear matter approach and then extended to ${ }_{\Lambda}^{12} \mathrm{C}$ via the local density approximation. In Refs. [41,42], a phenomenological scheme based on data on pion absorption in nuclei was used to predict the $n p$-induced decay rate $\Gamma_{n p}$. Ref. [42] updates the predictions of Ref. [41] by using more realistic $\Lambda$ wave functions in hypernuclei and short-range correlations in the baryon-baryon strong interactions. All charge channels of the two-nucleon stimulated decay, $\Lambda n n \rightarrow n n n, \Lambda n p \rightarrow n n p$ and $\Lambda p p \rightarrow n p p$, are included in the diagrammatic microscopic approach of Refs. [39,44-46]. In particular, Pauli exchange and ground state correlations were also studied $[39,46]$.

It is worthwhile to notice that the two values obtained by FINUDA are not independent of each other, since the proton required in coincidence with a $\pi^{-}$in Ref. [8] and with a $\left(\pi^{-}, n\right)$ pair in Ref. [9] belongs to the same experimental sample. To compare with the KEK-E508 determination [48], the FINUDA value affected by a smaller error [9], can be used: the two results are completely compatible within errors. 
Table 2

Kinematics of the three $\Lambda(n p) \rightarrow n n p$ candidate events: $p_{\pi^{-}}$indicates the momentum of the hypernucleus formation $\pi^{-}, T_{p}$ the $p$ kinetic energy, $T_{n 1}$ the $n$ kinetic energy of one of the neutrons, $T_{n 2}$ the kinetic energy of the second one, $\theta_{n 1 n 2}$ the angle between the two $n$ 's, $\theta_{n 1 p}$ the angle between the first $n$ and the $p, \theta_{n 2 p}$ the angle between the second $n$ and the $p, \theta_{\pi^{-}}$the angle between the formation $\pi^{-}$and the $p, p_{\text {miss }}$ the missing momentum of the final state, corresponding to the recoil momentum of the residual nucleus in a 4-body final state, and MM the missing mass for the decay of the hypernucleus from its ground state.

\begin{tabular}{llll}
\hline Target & ${ }^{7} \mathrm{Li}$ & ${ }^{7} \mathrm{Li}$ & ${ }^{9} \mathrm{Be}$ \\
\hline$p_{\pi^{-}}(\mathrm{MeV} / c)$ & $276.9 \pm 1.2$ & $276.5 \pm 1.2$ & $286.7 \pm 1.2$ \\
$T_{p}(\mathrm{MeV})$ & $51.11 \pm 0.85$ & $90.83 \pm 0.50$ & $71.77 \pm 0.80$ \\
$T_{n 1}(\mathrm{MeV})$ & $110 \pm 23$ & $21.0 \pm 2.0$ & $20.2 \pm 2.5$ \\
$T_{n 2}(\mathrm{MeV})$ & $16.9 \pm 1.7$ & $35.3 \pm 3.6$ & $31.5 \pm 4.2$ \\
$\theta_{n 1 n 2}(\mathrm{deg})$ & $94.8 \pm 3.8$ & $126.5 \pm 5.4$ & $133.6 \pm 7.5$ \\
$\theta_{n 1 p}(\mathrm{deg})$ & $102.2 \pm 3.4$ & $53.5 \pm 4.3$ & $128.5 \pm 5.5$ \\
$\theta_{n 2 p}(\mathrm{deg})$ & $154 \pm 19$ & $124.6 \pm 3.9$ & $95.4 \pm 3.6$ \\
$\theta_{\pi^{-}}(\mathrm{deg})$ & $33.4 \pm 3.7$ & $121.7 \pm 3.2$ & $159.3 \pm 5.9$ \\
$p_{\text {miss }}(\mathrm{MeV} / c)$ & $217 \pm 44$ & $447 \pm 18$ & $253 \pm 18$ \\
$\mathrm{MM}\left(\mathrm{MeV} / c^{2}\right)$ & $3710 \pm 23$ & $3720.3 \pm 4.7$ & $5617.3 \pm 5.0$ \\
\hline
\end{tabular}

\section{Direct experimental evidence of the $2 \mathrm{~N}$-induced NMWD}

Even though the assessment of the existence of $2 \mathrm{~N}$-induced weak decay, inferred from different experiments with different techniques, leads to the same results within the (large) errors, some skepticism could remain. The "smoking gun" proof, i.e. the measurement of a triple $(n, n, p)$ coincidence, in well defined and clean kinematics, of the three outgoing nucleons from the decay of an hypernucleus in its ground state is lacking.

Analyzing the full FINUDA data sample, $101\left(\pi^{-}, n, p\right)$ coincidence events have been found with the analysis selection on $E_{p}$ and $\cos \theta(n p)$ described in Section 4. This sample is well enriched (a factor $\sim 2.5$ ) in events due to the two-nucleon induced NMWD reaction, as outlined in the previous paragraph. Taking into account a $\Gamma_{2} / \Gamma_{N M}$ ratio value of 0.21 [9], the enrichment factor and the global neutron efficiency of the apparatus, it is expected to find $\sim 2.5[\Lambda+(n p) \rightarrow$ $n+n+p$ ] decay events. One event was found, in fact, satisfying the above requirements and with an additional neutral particle in the final state, on ${ }^{7} \mathrm{Li}$ target.

For this event the missing momentum is evaluated according to the hypothesis of a decay at rest of the hypernucleus and the missing mass of the reaction is calculated. The angular correlation among the three nucleons and between the $\pi^{-}$and proton is then considered to define exclusively the final state of the decay process. Table 2 reports, in column 2 , the kinematics while Fig. 5 displays this event into the $r-\phi$ plane of the apparatus.

The statistical uncertainties on the angles between the tracks (rows 6, 7, 8 and 9) have been evaluated with Gaussian error propagation taking into account the spatial resolution of the subdetectors. The event on ${ }^{7} \mathrm{Li}$ target, corresponding to the second column of Table 2 and to Fig. 5, displays a total kinetic energy of the three nucleons $T_{n 1 n 2 p}=178 \pm 23 \mathrm{MeV}$ and a missing mass $\mathrm{MM}=3710 \pm 23 \mathrm{MeV} / c^{2}$. In the first two columns of Table 3 the possible final state configurations following the $2 \mathrm{~N}$-induced decay of ${ }_{\Lambda}^{7} \mathrm{Li}$ to bound or partially bound residual nuclear systems are indicated, together with the corresponding expected missing mass. The configuration containing ${ }^{4} \mathrm{He}$ has the smallest missing mass due to the high binding energy of the nucleus $(\sim 28 \mathrm{MeV})$. The missing mass value measured in the event is compatible within the global resolution at the $0.76 \sigma$ level with the ${ }^{4} \mathrm{He}$ final state and within $(1.62-1.65) \sigma$ with the other two 


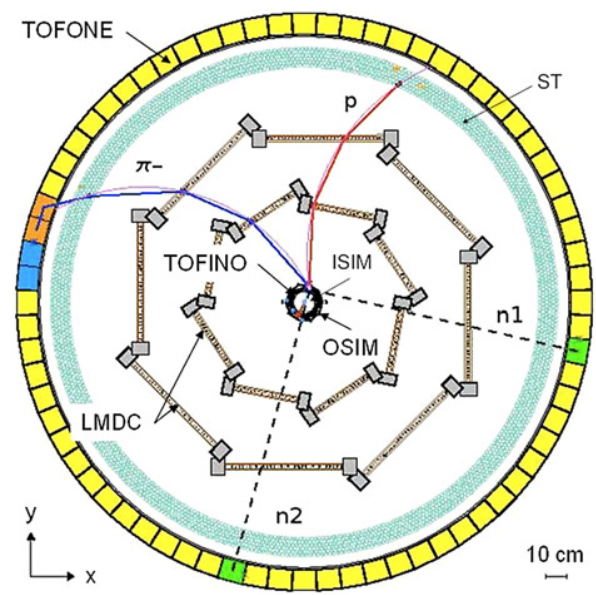

Fig. 5. Triple coincidence $\left(\pi^{-}, p, n, n\right)$ event coming from ${ }^{7} \mathrm{Li}$ target detected with the FINUDA spectrometer.

Table 3

Column one: undetected nuclear systems for the $2 \mathrm{~N}$-induced decay of ${ }_{\Lambda}^{7} \mathrm{Li}$; column two: missing mass with respect to the detected $(n 1, n 2, p)$ system; column three: undetected nuclear systems for the $2 \mathrm{~N}$-induced decay of ${ }_{\Lambda}^{9} \mathrm{Be}$; column four: missing mass with respect to the detected $(n 1, n 2, p)$ system.

\begin{tabular}{llll}
\hline${ }_{\Lambda}^{7} \mathrm{Li}$ & $\mathrm{MM}\left(\mathrm{MeV} / c^{2}\right)$ & ${ }_{\Lambda}^{9} \mathrm{Be}$ & $\mathrm{MM}\left(\mathrm{MeV} / c^{2}\right)$ \\
\hline${ }^{4} \mathrm{He}$ & 3727.4 & ${ }^{6} \mathrm{Li}$ & 5601.5 \\
${ }^{3} \mathrm{He}+n$ & 3748.0 & ${ }^{5} \mathrm{Li}+n$ & 5607.2 \\
${ }^{3} \mathrm{H}+p$ & 3747.2 & ${ }^{4} \mathrm{He}+d$ & 5603.0 \\
& & ${ }^{3} \mathrm{He}+{ }^{3} \mathrm{H}$ & 5617.3 \\
\hline
\end{tabular}

states, thus favouring the hypothesis of the first configuration. The topology of the event, with the neutron $n_{2}$ of $T_{n 2}=(16.9 \pm 1.7) \mathrm{MeV}$ kinetic energy at an angle of $(154 \pm 19)^{\circ}$ with respect to the proton of $T_{p}=(51.11 \pm 0.85) \mathrm{MeV}$, and the neutron $n_{1}$ of $T_{n 1}=(110 \pm 23) \mathrm{MeV}$ kinetic energy at an angle of $(102.2 \pm 3.4)^{\circ}$ with respect to the proton and an angle of $(94.8 \pm 3.8)^{\circ}$ with respect to $n_{2}$, introduces the possibility of a proton induced decay followed by a $n n$ scattering, for which the angle should be $90^{\circ}$ without considering the Fermi motion. However, for a 16.9 MeV kinetic energy scattered neutron to be emitted, the incident neutron should have an energy $\geqslant 130 \mathrm{MeV}$. In [8] the energy spectrum of protons coming from NMWD of ${ }_{\Lambda}^{7} \mathrm{Li}$ has been discussed and the contribution of the one-proton induced NMWD has been described by a Gaussian function centered at $76.7 \pm 5.2 \mathrm{MeV}$, with a standard deviation of $15.0 \pm 2.5 \mathrm{MeV}$. From this consideration, it is possible to exclude with a confidence level higher than $99 \%$ (normal test) that $n_{2}$ comes from a $n_{1} n_{2}$ rescattering process. We conclude that the event can be attributed to the exclusive ${ }_{\Lambda}^{7} \mathrm{Li} \rightarrow{ }^{4} \mathrm{He}+n+n+p$ reaction.

Triggered by this observation we looked at events in which no cut was imposed on the proton kinetic energy and we found $307\left(\pi^{-}, n, p\right)$ events. Asking in addition the presence of a neutral particle, detected in coincidence, two more events were found, one again from a ${ }^{7} \mathrm{Li}$ target and one from ${ }^{9} \mathrm{Be}$ target. Both $\left(\pi^{-}, n, n, p\right)$ events fulfill the request that the total kinetic energy of the three nucleons is $\leqslant 195 \mathrm{MeV}$ to fit with the Q-value of the $2 \mathrm{~N}$ decay reaction (kinematics in Table 2, columns 3 and 4). 


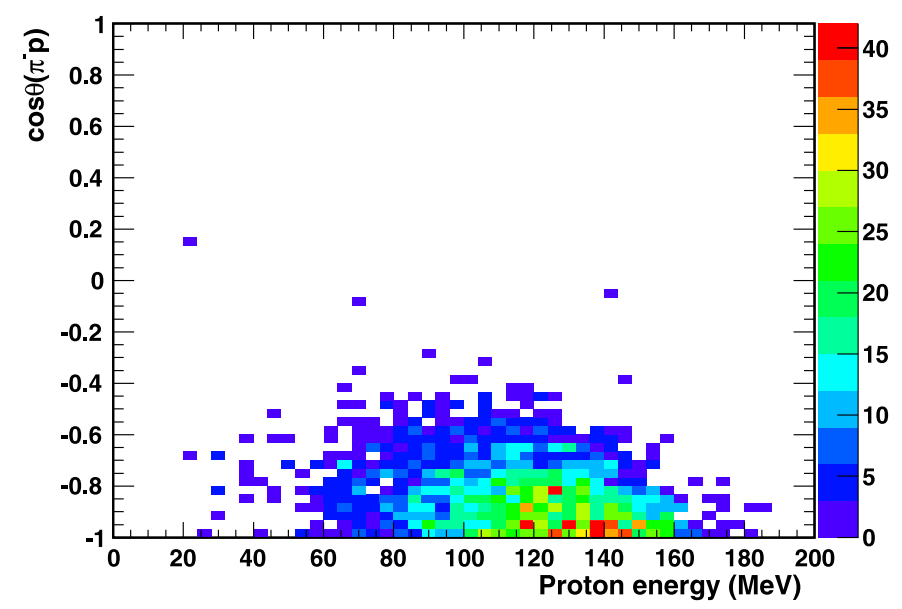

Fig. 6. Correlation between $\theta_{\pi^{-}} p$ and the proton energy for the simulated $K^{-}(n p) \rightarrow \Sigma^{-} p$ background events.

Extensive Monte Carlo simulations allowed to identify one source of background for these ( $\left.\pi^{-}, n, n, p\right)$ coincidences, due to events in which a one-proton induced decay is detected together with a $\gamma$ coming from the decay chain $K^{+} \rightarrow \pi^{+} \pi^{0}, \pi^{0} \rightarrow \gamma \gamma$, for which the $\pi^{+}$and the second $\gamma$ are not detected. Taking into account the detection efficiency for $\gamma$ 's with energy $\geqslant 70 \mathrm{MeV}$, for the $307\left(\pi^{-}, n, p\right)$ coincidence events $\sim 0.3$ spurious $\left(\pi^{-}, n, n, p\right)$ events are expected; so it is safe to consider the two observed events as free from that background.

Moreover, when selecting the $\pi^{-}$momentum to identify the formation of the hypernucleus in the ground state another background source appears in the same momentum range, due to the $K^{-}(n p) \rightarrow \Sigma^{-} p$ absorption on two correlated nucleons in the absorbing nucleus, followed by the in-flight $\Sigma^{-} \rightarrow \pi^{-} n$ decay. Events from this background show a significant back-to-back correlation; this feature rules out completely the first event discussed before. Considering the two additional events, the angle $\left(\theta_{\pi^{-}}\right)$between the $\pi^{-}$and $p$ was respectively $(121.7 \pm 3.2)^{\circ}$ and $(159.3 \pm 5.9)^{\circ}$, allowing them to be due to the $K^{-}(n p) \rightarrow \Sigma^{-} p$ background. This background reaction was simulated and the correlation between $\cos \left(\theta_{\pi^{-}}\right)$and the proton energy $E_{p}$ was studied; the result is reported in Fig. 6. It shows that the major contribution of this background channel is expected for $\pi^{-}$and $p$ emitted in a quasi back-to-back topology and for proton with $E_{p}$ larger then $\sim 100 \mathrm{MeV}$. In order to estimate the contribution of this reaction to the two $\left(\pi^{-}, n, n, p\right)$ additional events we evaluated the number of simulated events surviving to a $3 \sigma$ cut on $\cos \left(\theta_{\pi^{-}}\right)$and $E_{p}$ for the ${ }^{7} \mathrm{Li}$ and ${ }^{9} \mathrm{Be}$ target from which these events come. A value of the order of $10^{-3}$ events was found for both targets and using a Poisson statistics it is possible to state that the two $\Lambda n p \rightarrow n n p$ real events do not arise from background to a confidence level larger than $99 \%$.

In the second event on ${ }^{7} \mathrm{Li}$ target, corresponding to the third column of Table 2 and to Fig. 7 (on the left), a total kinetic energy of the three nucleons $T_{n 1 n 2 p}=147.1 \pm 4.2 \mathrm{MeV}$ and a missing mass $\mathrm{MM}=3720.3 \pm 4.7 \mathrm{MeV} / c^{2}$ have been measured. The missing mass value of the event is compatible within $1.5 \sigma$ with the ${ }^{4} \mathrm{He}$ final state and within (5.7-5.9) $\sigma$ with the other two states, clearly favouring a ${ }^{4} \mathrm{He}$ exclusive reaction. In this case, the angle between the proton having $T_{p}=90.83 \pm 0.50 \mathrm{MeV}$ and the neutron of $T_{n 1}=21.0 \pm 2.0 \mathrm{MeV}$ is $(53.5 \pm 4.3)^{\circ}$; the hypothesis that $n_{1}$ could come from a final state rescattering of the energetic proton can be rejected, being the difference between the masses of the two particles so small that angles sensibly different from 

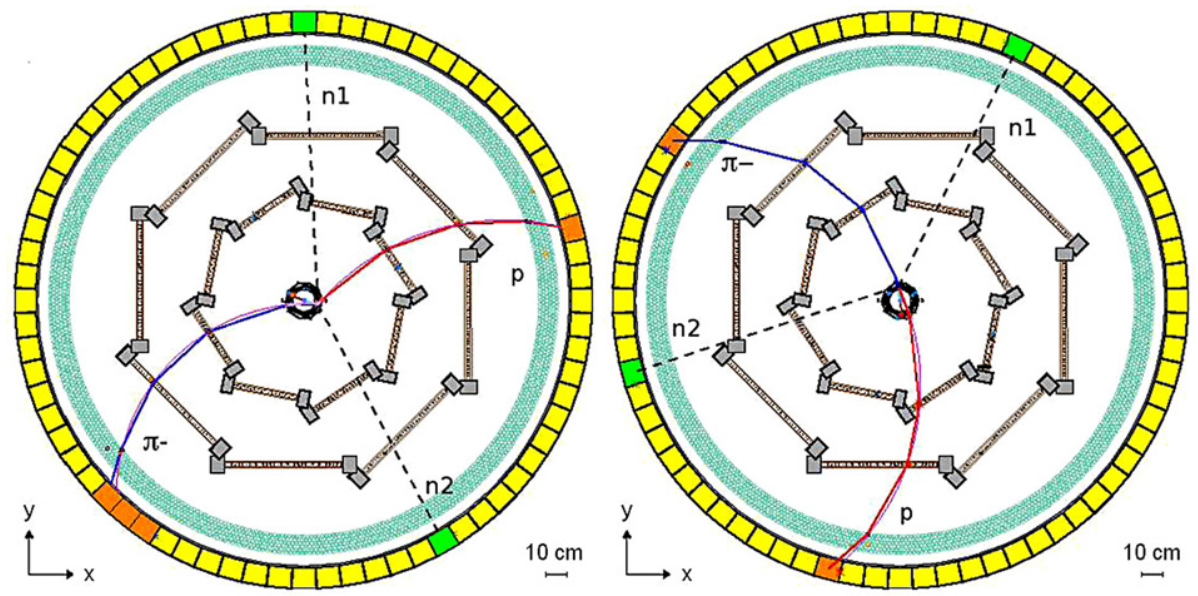

Fig. 7. Four-particle coincidence $\left(\pi^{-}, p, n, n\right)$ event coming from ${ }^{7} \mathrm{Li}$ target (on the left) and from ${ }^{9} \mathrm{Be}$ target (on the right) detected by FINUDA.

$90^{\circ}$ are not kinematically allowed. Therefore, this event also can be attributed to the exclusive ${ }_{\Lambda}^{7} \mathrm{Li} \rightarrow{ }^{4} \mathrm{He}+n+n+p$ reaction.

Finally, concerning the event on ${ }^{9} \mathrm{Be}$ target, corresponding to the fourth column of Table 2 and to Fig. 7 (on the right), a total kinetic energy of the three nucleons $T_{n 1 n 2 p}=123.5 \pm 4.9 \mathrm{MeV}$ and a missing mass $\mathrm{MM}=5617.3 \pm 5.0 \mathrm{MeV} / c^{2}$ have been obtained. Columns three and four of Table 3 list the possible final state configurations for the $2 \mathrm{~N}$-induced decay of ${ }_{\Lambda}^{9} \mathrm{Be}$ to bound or partially bound residual nuclear systems and the corresponding missing mass. The configuration with ${ }^{6} \mathrm{Li}$ is the only possible four body final state and has the smallest missing mass due to the binding energy of the ${ }^{6} \mathrm{Li}$ nucleus, even smaller than that of the configuration with ${ }^{4} \mathrm{He}$ due to the small binding energy of the partner deuteron; the mirror ${ }^{3} \mathrm{He}+{ }^{3} \mathrm{H}$ configuration features the largest missing mass value. The measured missing mass is compatible to less than $0.1 \sigma$, with the ${ }^{3} \mathrm{He}+{ }^{3} \mathrm{H}$ final state, to $2.1 \sigma$ with the ${ }^{5} \mathrm{Li}+n$ state, to $2.9 \sigma$ with the ${ }^{4} \mathrm{He}+d$ state and to $3.2 \sigma$ with the ${ }^{6} \mathrm{Li}$ final state. The kinematics of the event excludes the possibility of a one proton induced decay followed by a nucleon-nucleon scattering with a confidence level $>90 \%$ (normal test). In fact, the neutron $n_{2}$ with $T_{n 2}=(31.5 \pm 4.2) \mathrm{MeV}$ kinetic energy forms an angle of $(95.4 \pm 3.6)^{\circ}$ with the proton having $T_{p}=(71.77 \pm 0.80) \mathrm{MeV}$, while the neutron $n_{1}$ with $T_{n 1}=(20.2 \pm 2.5) \mathrm{MeV}$ kinetic energy forms an angle of $(128.5 \pm 5.5)^{\circ}$ with the proton and an angle of $(133.6 \pm 7.5)^{\circ}$ with $n_{2}$. Again, from the kinematic analysis, we conclude that the third event can be attributed to the exclusive ${ }_{\Lambda}^{9} \mathrm{Be} \rightarrow{ }^{3} \mathrm{He}+{ }^{3} \mathrm{H}+n+n+p$ reaction.

The three events discussed above represent the first direct experimental evidence of ( $n n p$ ) triplets following the NMWD of hypernuclei, very likely two-nucleon induced NMWD decays. From a quantum mechanical point of view it is not possible to disentangle two-nucleon induced NMWD events from spurious events attributable to FSI. However, the importance of the two contributions can be varied by selecting specific kinematical configurations. It is the case for the three discussed events. The probability that these events could be due to FSI effects may be deduced from the quoted errors, and has been evaluated for each event, as discussed before.

The exiguousness of the sample, however, prevents from making calculations of the $2 \mathrm{~N}$ induced NMWD rate. Some consideration can be put forward. ${ }_{\Lambda}^{7} \mathrm{Li}$ is a suitable hypernucleus to observe an exclusive $2 \mathrm{~N}$-induced decay since the structure of the system is a ${ }^{6} \mathrm{Li}$ core nucleus 
plus a $\Lambda$; moreover, it is well known $[49,50]$ that ${ }^{6} \mathrm{Li}$ can be understood as a quasi-molecule $(\alpha+d)$. Also ${ }_{\Lambda}^{9} \mathrm{Be}$ is a suitable hypernucleus to observe an exclusive $2 \mathrm{~N}$-induced decay since the structure of the system is a ${ }^{8} \mathrm{Be}$ core nucleus $(\alpha+\alpha)$ plus a $\Lambda$ [51]. The main difference between the decay mechanisms of ${ }_{\Lambda}^{7} \mathrm{Li}$ and ${ }_{\Lambda}^{9} \mathrm{Be}$ is related to the final state multiplicity: for ${ }_{\Lambda}^{7} \mathrm{Li}$ the residual nucleus is completely bound while for ${ }_{\Lambda}^{9} \mathrm{Be}$ the final configuration is composed by two mirror nuclear fragments. In [8] it was noticed that, for one-proton induced decays, a final nuclear state consisting of two or more nuclear fragments is more probable for larger mass numbers. This circumstance seems to be confirmed by the present observation of $2 \mathrm{~N}$-induced decay final states with a single residual nucleus for ${ }_{\Lambda}^{7} \mathrm{Li}$ and with two equivalent nuclear fragments for ${ }_{\Lambda}^{9} \mathrm{Be}$.

\section{Conclusions}

Thanks to its excellent instrumental capabilities, FINUDA was able to provide a substantial step forward in the weak decay study of $p$-shell hypernuclei. $\pi^{-}$decay rates and spectra were measured for the first time allowing the determination of the spin-parity of ${ }_{\Lambda}^{15} \mathrm{~N}\left(J^{\pi}=3 / 2^{+}\right)$. Proton spectra from NMWD were measured with an unprecedented resolution and low energy threshold. The branching ratio for the $\Lambda N N \rightarrow n N N$ was determined with an error of $33 \%$, the smallest so far. Finally three events, featuring all the three particle emitted in the $2 \mathrm{~N}$-induced NMWD $(n, n, p)$, were found and they can be quite safely attributed to this decay mode. They represent the first direct experimental evidence of this reaction.

The limitation of the above results is the low statistics that could be improved at the new JPARC machine; they could serve as a starting point for more precise and complete experiments. Improvements are needed on both the aspects of beam intensities and purity and of the design of novel detectors featuring new state-of-art technologies. Concerning the secondary beams $\left(\mathrm{K}^{-}\right.$or $\pi^{+}$), J-PARC is the answer, when the intensity of the proton beam in the slow extraction mode will reach a value close to the design one. Concerning the detectors, the recently approved construction of a new SKS-like spectrometer will be again the best tool to prepare $\Lambda$-hypernuclei in a defined initial state with both $\mathrm{K}^{-}$and $\pi^{+}$beams. A major effort is needed for the design and construction of new detector's arrays for measuring the weak decay products. Of big importance, but also of big difficulty, detectors for neutrons featuring complete angular coverage, good efficiency and good energy resolution. We think that the first requirement is the most important for a good mapping of the properties of both one nucleon and two-nucleon induced weak decays. As discussed, the interaction must be cleaned up as much as possible from the unavoidable effects of FSI. Systematic studies over some nuclei are necessary, as part of a coherent research program. Another instrumental aspect that could be looked for is the investigation of the advantages provided by gaseous detectors, eventually at low pressure, to detect some of the nuclear recoils produced in some processes, that could help in disentangling different hypotheses.

From the theoretical side, the predictions concerning MWD taking into account most of the effects (nuclear structure, bare interaction, etc.) are in good shape and just well confirmed by the recent data. Concerning NMWD, only the raw features were obtained, without detailed predictions on exclusive processes, in which also the final state is fully constrained. The calculations are indeed very hard, like the experiments, but something has to be started, just for stimulating the experimentalists to perform harder measurements.

\section{References}

[1] W. Cheston, H. Primakoff, Phys. Rev. 92 (1953) 1537. 
[2] W.M. Alberico, A. De Pace, M. Ericson, A. Molinari, Phys. Lett. B 256 (1991) 134.

[3] M.M. Block, R.H. Dalitz, Phys. Rev. Lett. 11 (1963) 96.

[4] W.M. Alberico, G. Garbarino, Phys. Rep. 369 (2002) 1.

[5] W.M. Alberico, G. Garbarino, in: T. Bressani, A. Filippi, U. Wiedner (Eds.), Hadron Physics, Proc. of the International School of Physics "E. Fermi" Course CLVIII, IOS Press, Amsterdam, 2005, p. 125.

[6] H. Outa, in: T. Bressani, A. Filippi, U. Wiedner (Eds.), Hadron Physics, Proc. of the International School of Physics

"E. Fermi" Course CLVIII, IOS Press, Amsterdam, 2005, p. 219.

[7] M. Agnello, et al., Phys. Lett. B 681 (2009) 139.

[8] M. Agnello, et al., Phys. Lett. B 685 (2010) 247.

[9] M. Agnello, et al., Phys. Lett. B 701 (2011) 556.

[10] M. Agnello, et al., Phys. Lett. B 698 (2011) 219.

[11] L. Celano, et al., Nucl. Instr. Meth. A 392 (1997) 304.

[12] M. Agnello, et al., Nucl. Phys. A 804 (2008) 151.

[13] E. Oset, P. Fernandez de Cordoba, J. Nieves, A. Ramos, L.L. Salcedo, Prog. Theor. Phys. Suppl. 117 (1994) 461.

[14] Y. Sato, et al., Phys. Rev. C 71 (2005) 025203.

[15] J. Sasao, et al., Phys. Lett. B 579 (2004) 258.

[16] J.J. Szymanski, et al., Phys. Rev. C 43 (1991) 849.

[17] S. Kameoka, et al., Nucl. Phys. A 754 (2005) 173c.

[18] A. Montwill, et al., Nucl. Phys. A 234 (1974) 413.

[19] H. Noumi, et al., Phys. Rev. C 52 (1995) 2936.

[20] T. Motoba, K. Itonaga, H. Bandō, Nucl. Phys. A 489 (1988) 683.

[21] T. Motoba, K. Itonaga, Prog. Theor. Phys. Suppl. 117 (1994) 477.

[22] A. Gal, Nucl. Phys. A 828 (2009) 72.

[23] S. Cohen, D. Kurath, Nucl. Phys. 73 (1965) 1;

S. Cohen, D. Kurath, Nucl. Phys. A 101 (1967) 1.

[24] D.J. Millener, Nucl. Phys. A 804 (2008) 84.

[25] M. Ukai, et al., Phys. Rev. C 77 (2008) 054315.

[26] O. Hashimoto, H. Tamura, Prog. Part. Nucl. Phys. 57 (2006) 564.

[27] M.M. Block, et al., in: Proceedings of the International Conference on Hyperfragments, St. Cergue, CERN64-1, 1964, p. 63.

[28] D. Bertrand, et al., Nucl. Phys. B 16 (1970) 77.

[29] D.H. Davis, R. Levi Setti, M. Raymund, Nucl. Phys. 41 (1963) 73;

R.H. Dalitz, Nucl. Phys. 41 (1963) 78.

[30] D. Ziemińska, Nucl. Phys. A 242 (1975) 461.

[31] D. Ziemińska, R.H. Dalitz, Nucl. Phys. A 238 (1975) 453;

D. Kiełczewska, D. Ziemińska, R.H. Dalitz, Nucl. Phys. A 333 (1980) 367.

[32] L. Tang, Int. J. Mod. Phys. E 10 (2010) 2638.

[33] S. Okada, et al., Nucl. Phys. A 754 (2005) 178c.

[34] S. Okada, et al., Phys. Lett. B 597 (2004) 249.

[35] A. Ramos, M.J. Vicente-Vacas, E. Oset, Phys. Rev. C 55 (1997) 735.

[36] A. Ramos, M.J. Vicente-Vacas, E. Oset, Phys. Rev. C 66 (2002) 039903.

[37] G. Garbarino, A. Parreño, A. Ramos, Phys. Rev. Lett. 91 (2003) 112501.

[38] G. Garbarino, A. Parreno, A. Ramos, Phys. Rev. C 69 (2004) 054603.

[39] E. Bauer, G. Garbarino, Nucl. Phys. A 828 (2009) 29.

[40] H. Bhang, et al., Eur. Phys. J. A 33 (2007) 259.

[41] A. Ramos, E. Oset, L.L. Salcedo, Phys. Rev. C 50 (1994) 2314.

[42] W.M. Alberico, A. De Pace, G. Garbarino, A. Ramos, Phys. Rev. C 61 (2000) 044314.

[43] E. Bauer, G. Garbarino, A. Parreño, A. Ramos, Nucl. Phys. A 836 (2010) 199.

[44] E. Bauer, F. Krmpotic, Nucl. Phys. A 739 (2004) 109.

[45] E. Bauer, Nucl. Phys. A 818 (2009) 174.

[46] E. Bauer, G. Garbarino, Phys. Rev. C 81 (2010) 064315.

[47] J.D. Parker, et al., Phys. Rev. C 76 (2007) 035501.

[48] M. Kim, et al., Phys. Rev. Lett. 103 (2009) 182502.

[49] H. Tamura, et al., Phys. Rev. Lett. 84 (2000) 5963.

[50] M. Agnello, et al., Nucl. Phys. A 775 (2006) 35.

[51] O. Hashimoto, et al., Nucl. Phys. A 639 (1998) 93c. 